DOI: 10.12957/demetra.2017.22483

\title{
Percepção da imagem corporal e estado nutricional em acadêmicas de nutrição de uma universidade pública
}

\section{Perception of body image and nutritional status in nutrition undergraduates from a public university}

Marcela Almeida Marreiros Lopes'

Adriana de Azevedo Paiva ${ }^{1,2}$

Sueli Maria Teixeira Lima'

Kyria Jayanne Clímaco Cruz ${ }^{1,2}$

Gilmara Péres Rodrigues'

Cecilia Maria Resende Gonçalves de

Carvalho,2

\section{Universidade Federal do Piauí. Centro de Ciências da Saúde. Departamento de Nutrição. Teresina-PI, Brasil. \\ ${ }^{2}$ Universidade Federal do Piauí. Centro de Ciências da Saúde. Departamento de Nutrição. Programa de Pós Graduação em Alimentos e Nutrição. Teresina-PI, Brasil.}

Correspondência / Correspondence

Adriana de Azevedo Paiva

E-mail: aapaiva@ufpi.edu.br

\section{Resumo}

Estudo transversal com 90 universitárias ingressantes (G1) e concluintes (G2) do curso de Nutrição, com objetivo de avaliar a associação entre a percepção da imagem corporal e o estado nutricional. Para avaliação da percepção e distorção da imagem corporal, aplicou-se Escala de Silhuetas de Stunkard e Questionário sobre Imagem Corporal (BSQ 34). Foi realizada aferição de peso $(\mathrm{Kg})$ e estatura $(\mathrm{m})$ e, a partir do cálculo do Índice de Massa Corporal (IMC), a classificação do estado nutricional. Os valores médios de peso, estatura e IMC foram semelhantes nos dois grupos $(\mathrm{p}>0,05)$, diferente do verificado para a idade, cuja média foi significativamente mais elevada entre as concluintes $(p<0,05)$. Constatou-se que $73,3 \%$ e $77,8 \%$ das acadêmicas ingressantes e concluintes, respectivamente, estavam eutróficas. Mas as concluintes apresentaram menor prevalência de baixo peso e excesso em relação às ingressantes, sem associação significativa $(p=0,87)$. A insatisfação por magreza ou excesso de peso foi de $62,3 \%$ e $82,2 \%$ no G1 e G2, respectivamente. Em relação à imagem corporal, observou-se que $20 \%$ das ingressantes mostraram distorção grave, com diferença expressiva quando comparadas às concluintes $(p=0,001)$. A insatisfação com a imagem corporal foi dominante, apresentando-se mais frequente entre as concluintes, que tinham uma impressão distorcida para o excesso de peso. A insatisfação com a imagem corporal em diferentes graus foi prevalente neste estudo, independente do estado nutricional. $\mathrm{O}$ incentivo à prática de exercício físico e a adoção de uma alimentação saudável devem ser encorajados em estudantes universitários, bem como a promoção de estratégias para uma percepção adequada do corpo. 
Palavras-chave: Imagem Corporal. Estado Nutricional. Estudantes.

\section{Abstract}

Cross-sectional study with 90 first-year (G1) and last-year undergraduates (G2) of the nutrition program, conducted to evaluate the association between body image perception and nutritional status. To evaluate the perception and distortion of body image, the Stunkard Silhouettes Scale and the Questionnaire on body image (BSQ 34) were applied. Weight $(\mathrm{Kg})$ and height $(\mathrm{m})$ were measured and nutritional status was classified based on the calculation of body mass index (BMI). The average values for weight, height and BMI were similar in both groups $(p>0.05)$, but a different result was found for age, whose average was significantly higher among graduates $(\mathrm{p}<0.05)$. It was found that $73.3 \%$ of the first-year students and $77.8 \%$ of the last-year undergraduates were eutrophic, and the latter had lower prevalence of underweight and overweight compared with the former, without a significant association $(\mathrm{p}=0.87)$. Dissatisfaction with thinness or overweight was $62.3 \%$ and $82.2 \%$ in G1 and G2, respectively. It was found that $20 \%$ of the first-year students showed serious body image distortion, with a significant difference when compared to last-year ones $(p=0.001)$. Body image dissatisfaction was quite prevalent, and it was more common among last-year students who had a distorted impression of excess weight. Body image dissatisfaction in different degrees was prevalent in this study, regardless of nutritional status. Physical exercise and healthy eating habits should be encouraged among college students, and strategies should be promoted for a better perception of one's body.

Keywords: Body Image. Nutritional Status. Perception. Students.

\section{Introdução}

O padrão estético atual supervaloriza a magreza. Assim, o corpo magro, definido e musculoso é indicativo social de saúde, beleza e poder. Esta realidade cria uma situação de frustração, diminuição da autoestima e discriminação aos que não se adaptam, favorecendo uma condição propícia para distorções na percepção da imagem corporal. ${ }^{1}$

A imagem corporal é um componente da identidade pessoal, podendo ser definida como a representação mental que uma pessoa tem sobre sua aparência física e corporeidade e é expressa 
por meio de pensamentos, sentimentos e comportamentos referentes ao corpo. Sua formação está relacionada à percepção corporal e ao nível de satisfação ou insatisfação com o próprio corpo. ${ }^{2}$

Enquanto alguns estudos ${ }^{3-5}$ sugerem que indivíduos com excesso de peso apresentam maior insatisfação com a imagem corporal, outros demonstram que, mesmo entre os que apresentam valores de índice de massa corporal adequado, a prevalência de insatisfação é elevada. ${ }^{6-7}$ Dessa forma, evidencia-se a necessidade de verificar a relação entre insatisfação com a imagem corporal e estado nutricional, na perspectiva de promover melhoras na percepção e aceitação do próprio corpo.

O período de transição de vida no final da adolescência, quando indivíduos começam a se preparar para a universidade ou uma vida de forma independente, é de suma importância no estabelecimento de comportamentos e na aquisição de hábitos que irão influenciar o potencial ganho de peso. ${ }^{8}$ Acadêmicas de nutrição estão em contato constante com o conhecimento sobre alimentação e nutrição e supõe-se que tenham adquirido informações que direcionem suas práticas para a obtenção de saúde, bem-estar e qualidade de vida. Diante disso, sugere-se que estudantes de nutrição estão em um ambiente que favorece a preocupação com a imagem corporal, gerando, em alguns casos, distorções e insatisfações com a autoimagem. ${ }^{1}$

É fundamental considerar que os futuros nutricionistas estarão, em sua vida profissional, orientando pessoas em relação à alimentação para obter resultados que se expressam na corporeidade e, dessa forma, sentir-se-ão sujeitos a expectativas sociais e expostos a críticas sobre seu peso e sua forma física. É imprescindível que tenham uma boa relação pessoal com a alimentação e o corpo para conseguir uma eficaz orientação na prática profissional. ${ }^{2}$

Na perspectiva de ampliar essa discussão, o presente estudo teve por objetivo avaliar a associação entre a percepção da imagem corporal e o estado nutricional em acadêmicas de nutrição, iniciantes e ingressantes, de uma universidade pública.

\section{Métodos}

Estudo transversal realizado entre 2014 e 2015 com estudantes do curso de Graduação em Nutrição da Universidade Federal do Piauí (UFPI), distribuídos em dois grupos segundo o período que cursavam: ingressantes $(\mathrm{G} 1, \mathrm{n}=45)$ e concluintes $(\mathrm{G} 2, \mathrm{n}=45)$. O tamanho da amostra foi obtido por meio de análise probabilística, a partir do universo de 174 alunas matriculadas nos

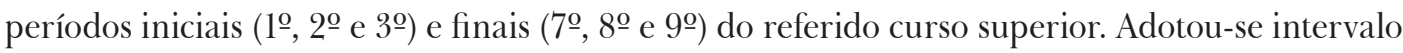
de confiança de $95 \%$ e margem de erro de $7,2 \%$.

Foram incluídas na pesquisa estudantes do sexo feminino, com idade superior a 19 anos, regularmente matriculadas nos períodos iniciais e finais do curso de Nutrição, no ano de 2015, excluindo-se gestantes, nutrizes, deficientes físicos, atletas e aquelas que estavam em acompanhamento nutricional. 
Inicialmente, foi conduzido um estudo piloto com $10 \%$ do universo de alunas $(n=17)$ da mesma faixa etária para testar os instrumentos de coleta dos dados. Para avaliar a percepção da imagem corporal, aplicou-se a Escala de Silhuetas de Stunkard, ${ }^{9}$ validada no Brasil por Scagliusi et al. ${ }^{10}$ Esse instrumento é composto por um conjunto de figuras humanas numeradas de 1 a 9 , que representam desde a magreza (silhueta 1) até a obesidade severa (silhueta 9). As estudantes indicaram a figura que melhor representava sua silhueta atual, bem como a que indicava a aparência desejada. A insatisfação com a imagem corporal foi identificada a partir da subtração entre os valores das silhuetas atual e desejada. Valores positivos indicaram insatisfação por excesso de peso e valores negativos, insatisfação por magreza, enquanto valores iguais a zero, satisfação.

A presença e o grau de insatisfação com a forma do corpo foram avaliados pelo Questionário sobre Imagem Corporal (Body Shape Questionnaire - BSQ 34), desenvolvido por Cooper et al. ${ }^{11} \mathrm{e}$ adaptado para o Brasil por Cordás \& Neves. ${ }^{12}$ Esse questionário apresenta 34 questões, cada uma com seis possibilidades de resposta, variando de "sempre" a "nunca", com pontuações entre 1 e 6. O resultado é obtido a partir do somatório das pontuações atribuídas a cada questão, classificado em quatro categorias que definem o nível de preocupação com a imagem corporal: (a) normalidade (menor que 70 pontos) ou ausência de distorção da imagem corporal; (b) leve distorção (entre 70 e 90 pontos); (c) moderada distorção (entre 91 e 110 pontos); e (d) grave distorção (acima de 110 pontos).

O estado nutricional foi classificado por aferição das medidas antropométricas, peso (kg) e estatura (m). Utilizou-se balança portátil digital, marca Plenna ${ }^{\circledR}$, com capacidade de $150 \mathrm{~kg}$ e sensibilidade de $50 \mathrm{~g}$, bem como fita métrica inelástica com $150 \mathrm{~cm}$ de comprimento. O índice de massa corporal (IMC em $\mathrm{kg} / \mathrm{m}^{2}$ ) foi calculado pela equação de Quetelet (IMC = Peso/Estatura ${ }^{2}$ ) e o diagnóstico nutricional baseou-se nos pontos de corte padronizados pela Organização Mundial da Saúde. ${ }^{13}$

A análise estatística foi realizada no SPSS 18.0 para Windows ${ }^{\circledR}$ (SPSS INC., Chicago, IL, EUA), por meio de estatística descritiva e analítica, considerando-se significativos valores de $\mathrm{p}<0,05$, com margem de confiança de 95\%. Aplicou-se teste de Kolmogorov-Smirnov, a fim de verificar a normalidade dos dados quantitativos, o teste $t$ de Student e Mann-Whitney, para comparação de médias entre os grupos, para variáveis simétricas e assimétricas, respectivamente, e o Teste de Fisher, para averiguar a associação entre as variáveis.

Esta pesquisa foi conduzida após aprovação pelo Comitê de Ética em Pesquisa (CEP) da Universidade Federal do Piauí, sob protocolo no 42010015.9.0000.5214 e após a assinatura do Termo de Consentimento Livre e Esclarecido pelas participantes, conforme prevê a Resolução 466/2012 do Conselho Nacional de Saúde. ${ }^{14}$ 


\section{Resultados}

O presente estudo avaliou 90 estudantes do sexo feminino do início e final do curso de Nutrição, com média (desvio-padrão) de idade de 21,4 (₫1,73) anos, com idade mínima de 20 anos e máxima de 30 anos. A tabela 1 apresenta os valores (média e desvio-padrão) da idade, peso corporal, estatura e índice de massa corpórea (IMC) das acadêmicas do curso de Nutrição investigadas. Observou-se que as médias de peso corporal, estatura e IMC foram semelhantes nos dois grupos pesquisados $(\mathrm{p}>0,05)$, diferente do verificado para a idade, cuja média foi significativamente mais elevada entre as acadêmicas concluintes $(\mathrm{p}<0,05)$.

Tabela 1. Valores médios e desvios-padrão da idade, peso corporal, estatura e índice de massa corpórea das acadêmicas do curso de Nutrição. Teresina-PI, 2014/2015.

\begin{tabular}{cccc}
\hline VARIÁVEL & $\begin{array}{c}\text { G1 (Ingressantes) } \\
(\mathrm{n}=45)\end{array}$ & $\begin{array}{c}\text { G2 (Concluintes) } \\
(\mathrm{n}=45)\end{array}$ & $p$ \\
Média $\pm \mathrm{DP}$ & Média $\pm \mathrm{DP}$ & \\
\hline Idade (anos) & $\mathrm{a}$ & $22,60 \pm 1,85$ & $<0,001^{*}$ \\
Peso corporal $(\mathrm{kg})^{\mathrm{a}}$ & $54,91 \pm 9,11$ & $54,91 \pm 9,16$ & 0,808 \\
Estatura $(\mathrm{m})^{\mathrm{a}}$ & $1,60 \pm 0,11$ & $1,62 \pm 0,05$ & 0,256 \\
IMC $\left(\mathrm{kg} / \mathrm{m}^{2}\right)^{\mathrm{b}}$ & $21,10 \pm 3,05$ & $20,83 \pm 2,89$ & 0,669 \\
\hline
\end{tabular}

IMC: Índice de Massa Corporal. ${ }^{\mathrm{a}}$ Teste Mann-Whitney; ${ }^{\mathrm{b}}$ Teste $\mathrm{t}(* \mathrm{p}<0,05)$

Na figura 1, nota-se que, de acordo com a classificação do estado nutricional, 73,3\% e 77,8\% das acadêmicas ingressantes (G1) e concluintes (G2), respectivamente, estavam eutróficas, mas as universitárias concluintes apresentaram menor prevalência de baixo peso e excesso de peso em relação às alunas iniciantes, porém, sem associação significativa entre os grupos $(p=0,87)$. 


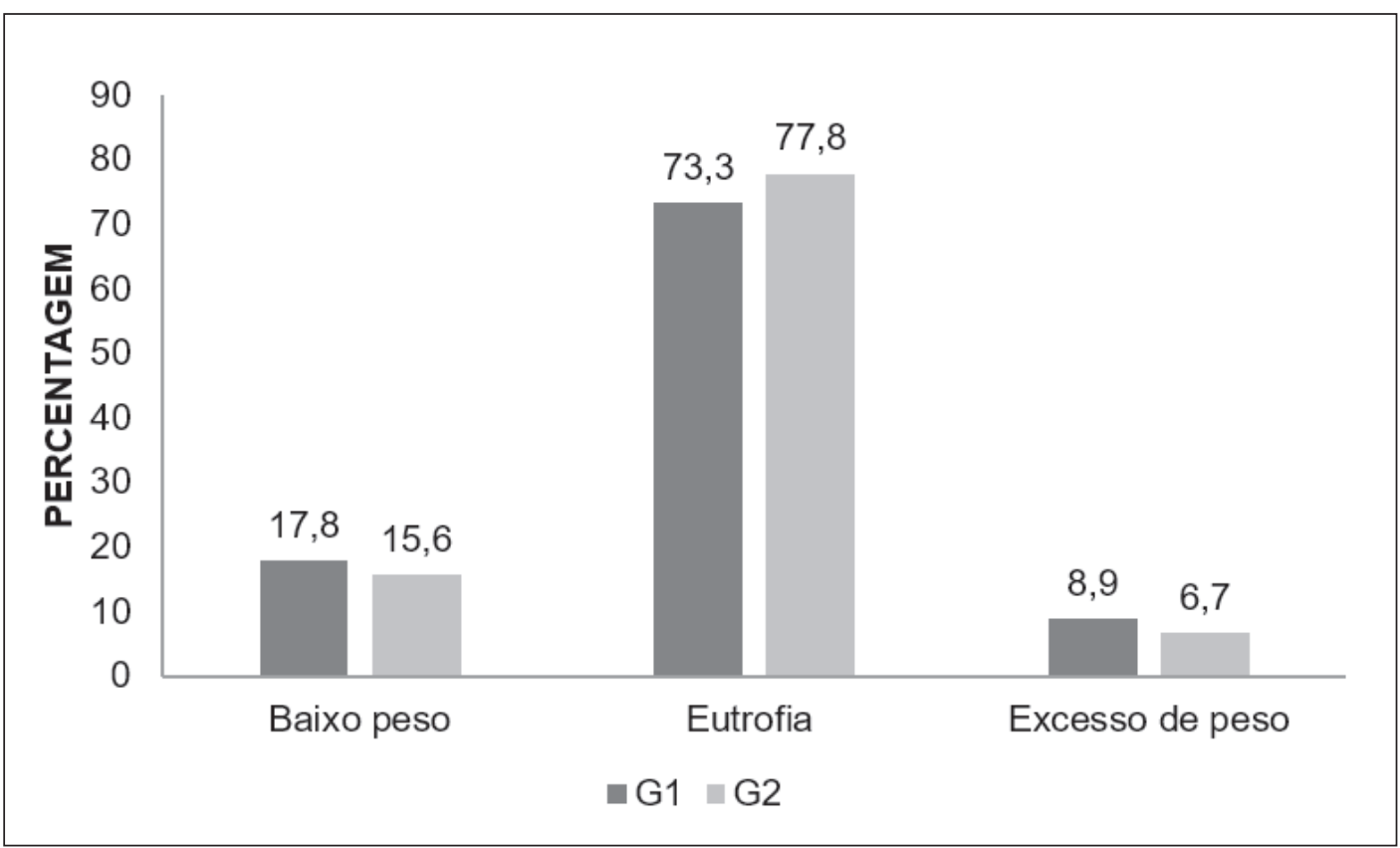

G1: acadêmicas ingressantes; G2: acadêmicas concluintes. Teste $\chi^{2}(p=0,87)$.

Figura 1. Distribuição porcentual das estudantes segundo estado nutricional. Teresina-PI, $2014 / 2015$.

A figura 2 mostra a distribuição porcentual segundo a satisfação/insatisfação com a imagem corporal das estudantes. Percebe-se porcentual elevado de insatisfação por magreza ou excesso de peso entre as estudantes avaliadas, sendo 62,3\% e 82,2\% nos grupos ingressantes (Gl) e concluintes (G2), respectivamente. 


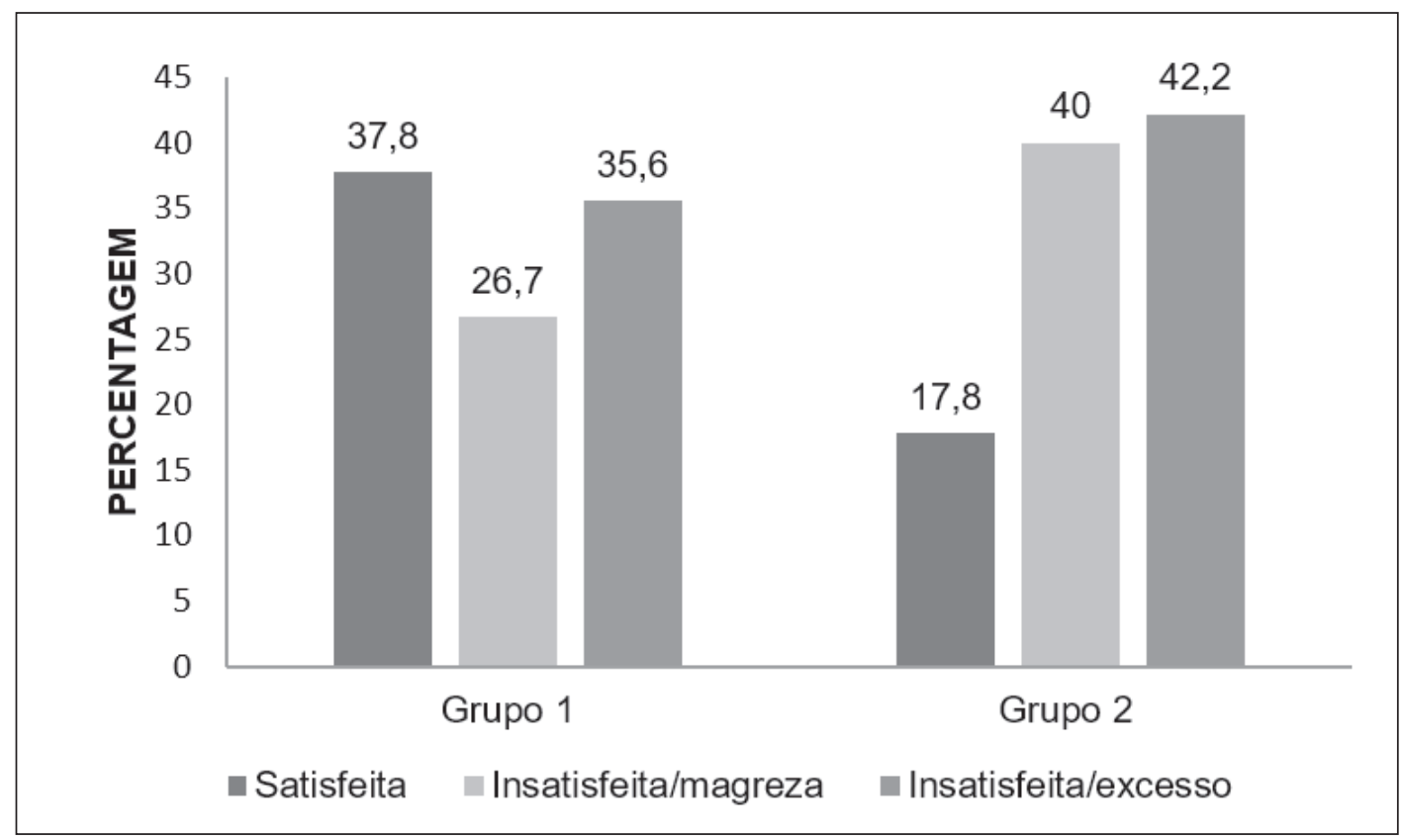

G1: acadêmicas ingressantes; G2: acadêmicas concluintes. Teste $\chi^{2}(p=0,096)$.

Figura 2. Distribuição porcentual das estudantes segundo satisfação com a imagem corporal. Teresina-PI, 2014/2015.

Em relação à imagem corporal, observou-se na figura 3 que $20 \%$ das acadêmicas do ano inicial do curso mostraram distorção grave, com diferença significativa quando comparadas às estudantes do final do curso $(\mathrm{p}=0,001)$.

A associação entre a percepção da imagem corporal e o estado nutricional está apresentada na tabela 2. A insatisfação com a imagem corporal foi prevalente entre as alunas avaliadas, independente do período em que se encontravam no curso e se o estado nutricional era adequado ou inadequado, portanto, não houve associação entre essas variáveis. 


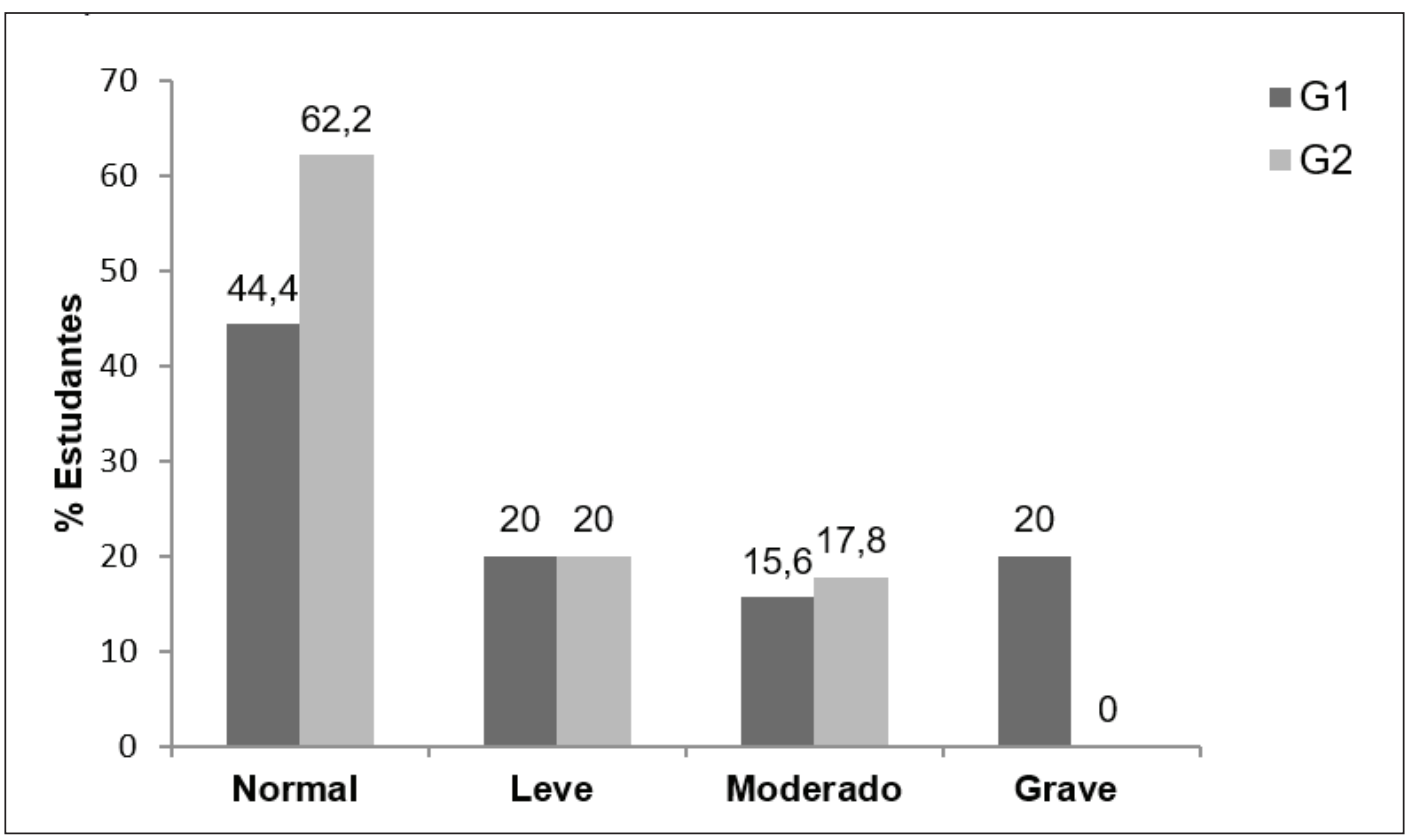

G1: ingressantes; G2: concluintes. Grau de distorção da imagem corporal G1 x G2: normal (x2=0,069); leve $(\mathrm{x} 2=0,604)$; moderado $(\mathrm{x} 2=0,500)$; grave $(\mathrm{x} 2=0,001)$.

Figura 3. Distribuição porcentual das estudantes segundo grau de distorção da imagem corporal. Teresina-PI, 2014/2015.

Tabela 2. Estado nutricional e satisfação com a imagem corporal das acadêmicas de Nutrição, conforme período de estudo no curso. Teresina-PI, 2014/2015.

\begin{tabular}{|c|c|c|c|c|}
\hline \multirow[b]{2}{*}{ Grupos } & \multirow{2}{*}{$\begin{array}{c}\text { Estado } \\
\text { Nutricional }\end{array}$} & \multicolumn{2}{|c|}{ Escala de silhuetas } & \multirow[b]{2}{*}{$p^{*}$} \\
\hline & & $\begin{array}{c}\text { Satisfeita } \\
\text { n }(\%)\end{array}$ & $\begin{array}{c}\text { Insatisfeita } \\
\text { n (\%) }\end{array}$ & \\
\hline \multirow{2}{*}{$\begin{array}{c}\text { G1 } \\
n=45\end{array}$} & Adequado & $14(42,4)$ & $19(57,6)$ & \multirow{2}{*}{0,488} \\
\hline & Inadequado & $3(25,0)$ & $9(75,0)$ & \\
\hline G2 & Adequado & $7(20,0)$ & $28(80,0)$ & \multirow{2}{*}{0,661} \\
\hline $\mathrm{n}=45$ & Inadequado & $1(10,0)$ & $9(90,0)$ & \\
\hline
\end{tabular}

G1: acadêmicas ingressantes; G2: acadêmicas concluintes. *valor de p do teste de Fisher. 


\section{Discussão}

Neste estudo foi avaliada a associação entre a percepção da imagem corporal e o estado nutricional em acadêmicas de nutrição. A maioria da população estudada apresentou eutrofia. No entanto, observaram-se porcentuais consideráveis de inadequações de peso, predominando o baixo peso, tanto em ingressantes quanto em concluintes, superando porcentuais encontrados por Silva et al., ${ }^{1}$ que, ao avaliarem 175 estudantes do curso de Nutrição da Universidade Federal de Ouro Preto (MG), notaram prevalência de 9,4\% de baixo peso e 8,5\% de excesso de peso.

Alguns estudos apontam que inadequações nutricionais são comuns entre estudantes universitárias, devido aos hábitos alimentares serem fortemente influenciados por fatores como o ingresso na universidade, falta de tempo para realizar refeições completas por causa das atividades acadêmicas, substituição de refeições completas por lanches práticos e rápidos e o estabelecimento de novos comportamentos e relações sociais, influenciando o excesso de peso e a distorção de imagem corporal..$^{5-17}$

A prevalência da obesidade feminina é preocupante e muitos fatores estão envolvidos nesse fenômeno, destacando-se as práticas alimentares inadequadas e o baixo nível de atividade física ${ }^{18}$ que acometem as mulheres brasileiras.

A obesidade traz prejuízos imensuráveis à saúde, seguida de alterações psicológicas e sociais significativas, como a percepção negativa do próprio corpo, que se expressa no cultivo a um ideal de corpo esbelto. ${ }^{18}$ Nesse sentido, ao analisar a percepção que as participantes têm sobre seu próprio corpo, observou-se elevada prevalência de insatisfação, seja por excesso de peso ou por magreza, especialmente entre as concluintes. Presume-se que esse resultado possa ser justificado pela maior cobrança com a aparência física em relação ao corpo ideal, especialmente por estarem próximas ao final do curso, quando necessitam ser exemplo aos futuros pacientes.

Sobre esse aspecto, Magalhães, ${ }^{2}$ ao investigar os fatores psicossociais relacionados à imagem corporal em estudantes de nutrição, constatou, na análise dos depoimentos das alunas concluintes, que a escolha pelo curso também foi baseada na preocupação com o peso corporal e expectativa de emagrecimento, busca da autoestima e aprovação social. Dessa forma, verifica-se que a pressão social em relação a sua forma física é considerável, sendo atribuído à magreza o significado de capacidade técnica e profissional.

É oportuno mencionar que uma boa percepção da imagem corporal é importante, pois está relacionada à autoestima do indivíduo, ou seja, satisfação pessoal e estar bem consigo mesmo. Ademais, a insatisfação corporal reflete-se na sua autoimagem, podendo levar o indivíduo a adotar condutas de saúde menos saudáveis para se enquadrar em uma imagem que acredita ser ideal. ${ }^{19}$ 
Magalhães, ${ }^{2}$ ao analisar a satisfação com a imagem corporal em estudantes de nutrição, constatou $80 \%$ e $78 \%$ de insatisfação corporal entre os ingressantes e concluintes, respectivamente. Destaca-se que, independente da condição de ingressante ou concluinte, a maioria das acadêmicas demostrou estar insatisfeita. Mais recentemente, Wanden-Berghe et al., ${ }^{20} \mathrm{em}$ seu estudo com universitárias, observaram insatisfação com imagem corporal em menor proporção $(41,1 \%)$, pois os universitários dessa pesquisa, além de preocupados com seu estado nutricional, seguiam dietas para perder peso.

$\mathrm{Na}$ análise da distorção da imagem corporal, percebeu-se que, em ambos os grupos, houve maior prevalência de normalidade, ou seja, ausência de distorção. No entanto, é importante ressaltar o porcentual elevado de estudantes ingressantes que apresentaram distorção grave da imagem do seu corpo. Esse porcentual é um dado preocupante, tendo em vista que elas serão futuras nutricionistas que também deverão atentar para a detecção e o manejo de comportamentos alimentares de risco em seus pacientes. Por outro lado, é provável que o conhecimento adquirido durante o curso possa ter influenciado de forma positiva a percepção mais adequada da imagem corporal, uma vez que estudos mostram que o conhecimento em nutrição pode ser determinante na subjetividade da imagem corporal. ${ }^{17-21}$

Silva et al.,' ao investigar a percepção corporal em acadêmicas de nutrição, demostraram que 63,4\% não tinham distorção, 22,9\% apresentaram distorção leve, $8,0 \%$, distorção moderada, e apenas $5,7 \%$ relataram distorção grave da imagem corporal. Bosi et al., ${ }^{5}$ ao avaliar o grau de distorção da imagem corporal, também constataram que a maioria não tinha distorção (59,6\%). Nilson et al., ${ }^{22}$ ao estudar a autoimagem corporal em estudantes universitários, mostraram percepção normal em $62,5 \%$, distorção leve em 20,8\% e moderada em $16,7 \%$, sendo estes porcentuais menores atribuídos ao fato de a maioria dos universitários serem homens, que, segundo a literatura, têm menor índice de insatisfação corporal quando comparada às mulheres. ${ }^{23}$

A análise de associação entre a percepção da imagem corporal e o estado nutricional demonstrou que, apesar de as acadêmicas concluintes apresentarem estado nutricional adequado, estavam insatisfeitas com sua imagem, sem associação entre essas variáveis, o que pode ser devido às possíveis implicações dos instrumentos utilizados.

Apesar de ser bem aceito a aplicação do método de silhuetas, estudos mostram que a avaliação da percepção da imagem por este método pode ser um fator limitante, porque usa uma figura bidimensional em preto e branco, que pode dificultar uma identificação mais apurada da percepção da imagem corporal. ${ }^{24,25}$ No entanto, a escolha da Escala de Silhuetas de Stunkard ${ }^{9}$ justifica-se pela sua utilização em inúmeros estudos e já ter sido validada no Brasil.

A partir desse resultado, pôde-se constatar que as universitárias concluintes do curso de nutrição, mesmo apresentando valores de IMC adequados, têm o desejo de reduzir ou aumentar 
o tamanho da silhueta, aproximando-se do estereótipo idealizado pela sociedade, o que poderia favorecer melhora na satisfação dessas estudantes com a imagem corporal.

Segundo Silva et al., ${ }^{1}$ universitárias eutróficas e insatisfeitas com a sua imagem corporal mostram uma preocupação exagerada com a estética do corpo. Os autores citam a importância da abordagem sobre o tema nos cursos de nutrição na perspectiva de alertar sobre a forte pressão sociocultural que impõe um ideal de corpo bonito e saudável, sobrepondo-se aos princípios da saúde e da atuação do profissional nutricionista.

Sobre este aspecto, outros estudos convergem com os resultados obtidos. Ferrari et al. ${ }^{6}$ observaram que a insatisfação com a imagem corporal está associada ao estado nutricional, sendo os universitários com excesso de peso os mais insatisfeitos. Coqueiro et al. ${ }^{3}$ notaram associação significativa entre estado nutricional e insatisfação com a imagem corporal, indicando que o IMC, parâmetro utilizado nesse estudo, não reflete a forma como os indivíduos percebem seus corpos.

Alvarenga et al. ${ }^{26}$ constataram que, em estudantes universitárias eutróficas, a insatisfação corporal é expressiva, pois escolhiam como ideal e saudável números menores que a figura atual (na escala de silhuetas). Os autores ressaltam que o fato de as eutróficas desejarem ter um corpo mais magro conduz a uma discussão sobre o conceito de saúde e boa forma vigente, associado a uma magreza não saudável de acordo com os parâmetros médicos.

A partir das reflexões levantadas, é preciso destacar que, apesar de a maioria das universitárias se encontrarem eutróficas, houve prevalência elevada de baixo peso e de excesso. Ademais, muitas referiram insatisfação com seu peso, seja por magreza ou por excesso de peso, de modo a se perceber que as estudantes estão desconfortáveis com seu corpo e em conflito consigo mesma, provavelmente pela busca de um padrão corporal valorizado como ideal, uma exigência imposta à mulher, pela mídia e pela sociedade.

No estudo da distorção da imagem corporal, embora a maioria tenha apresentado normalidade, nas ingressantes, obteve-se $20 \%$ de distorção grave. Ao verificar a associação entre a percepção da imagem corporal e o estado nutricional, observou-se que a insatisfação foi bastante dominante, com mais frequência entre as concluintes, que apresentavam uma impressão distorcida para o excesso de peso.

Diante disso, verifica-se que a propagação de estereótipos magros tem influenciado cada vez mais a busca por esses padrões, principalmente entre os indivíduos com excesso de peso. Dessa forma, esse fato deve ser utilizado como indicação para a promoção de mudanças no estilo de vida que visem à adequação a um estado nutricional mais saudável, promovendo, também, mudanças positivas na imagem corporal. ${ }^{6}$ 


\section{Conclusão}

Neste estudo, observou-se maior prevalência de baixo peso e excesso de peso nas estudantes ingressantes em relação às concluintes. A insatisfação com a imagem corporal em diferentes graus foi elevada e apresentou-se mais frequente entre as concluintes, especialmente para o grupo com excesso de peso. Não houve associação significativa entre a imagem corporal e o estado nutricional.

Esses achados reforçam a necessidade de outros estudos que aprofundem essas questões no ambiente universitário, com o objetivo de incentivar a adoção de uma alimentação saudável e a prática de exercício físico, sobretudo para as estudantes com sobrepeso, obesidade ou baixo peso.

A universidade precisa promover e apoiar programas para estimular os estudantes a cuidar da saúde, obviamente procurando encontrar a melhor maneira de atuar para o bem-estar e a qualidade de vida dos universitários. É preciso entender que a imagem corporal pode ser distorcida entre as estudantes, dessa forma, o envolvimento de todos como agente ativo na promoção de estratégias para uma percepção mais adequada do corpo produzirá efeitos significativos, além de promover a perda de peso e prevenir a obesidade para o alcance de uma vida longa e mais saudável.

\section{Colaboradores}

Lopes MAM participou de todas as etapas da pesquisa, incluindo o levantamento bibliográfico e a redação do manuscrito; Paiva AA, atuou na revisão contribuiu na análise, interpretação dos dados e revisão crítica do texto; Lima SMT, na interpretação dos dados e revisão crítica do texto; Cruz KJC foi responsável pela realização dos testes estatísticos; Rodrigues GP participou no planejamento e organização da pesquisa e contribuiu na revisão do artigo; de Carvalho CMRG contribuiu no desenho e concepção da pesquisa, análise e interpretação dos dados e revisão final do artigo.

Conflito de Interesses: Os autores declaram não haver conflito de interesses.

\section{Referências}

1. Silva JD, Silva ABJ, Oliveira AVK, Nemer ASA. Influência do estado nutricional no risco para transtornos alimentares em estudantes de nutrição. Ciênc Saúde Coletiva 2012; 17(12):3399-3406.

2. Magalhães P. Comportamento alimentar, estado nutricional e imagem corporal de estudantes de nutrição: aspectos psicossociais e percurso pedagógico [tese]. [Araraquara, SP]: Faculdade de Ciências Farmacêuticas; 2011.

3. Coqueiro RS, Petroski EL, Pelegrini A, Barbosa AR. Insatisfação com a imagem corporal: avaliação comparativa da associação com estado nutricional em universitários. Rev Psiquiatr. 2008; 30(1):31-38. 
4. Kakeshita IS, Almeida SS. Relação entre índice de massa corporal e a percepção da auto imagem em universitários. Rev. Saúde Públ. 2006; 40(3):497-504.

5. Bosi MLM, Luiz RR, Morgado CMC, Costa MLS, Carvalho RJ. Auto percepção da imagem corporal entre estudantes de nutrição: um estudo no município do Rio de Janeiro. J. Bras. Psiquiat. 2006; 55(2):108-113.

6. Ferrari EP, Gordia AP, Martins CR, Silva DA, Quadros TM, Petroski EL. Insatisfação com a imagem corporal e relação com o nível de atividade física e estado nutricional em universitários. Motricidade 2012; 8(3):52-58.

7. Rech CR, Araújo EDS, Vanat JR. Autopercepção da imagem corporal em estudantes do curso de educação física. Rev Bras Educ Fís Esporte 2010; 24(2):285-292.

8. Yang K, Turk MT, Allison LV, James KA, Chasens E. Body mass index self-perception and weight management behaviors during late adolescence. J. School Health 2014; 84(10):654-660.

9. Stunkard AJ, Sorensen T, Schulsinger F. Use of the Danish adoption register for the study of obesity and thinness. In: Kety SS, Rowland LP, Sidman RL, Mathysse SW, editores. The genetics of neurological and psychiatric disorders. Nova Iorque: Raven Press; 1983. p. 115-120.

10. Scagliusi FB, Polacow VO, Cordás TA, Coelho D, Alvarenga M, Philippi ST, et al. Tradução, adaptação e avaliação psicométrica da Escala de Conhecimento Nutricional do National Health Interview Survey Cancer Epidemiology. Rev Nutr. 2006; 19(4):425-436. .

11. Cooper PJ, Taylor M, Cooper Z, Fairburn CG. The development and validation of the Body Shape Questionnaire. Int J Eat Disord. 1987; 6:485-94.

12. Cordás TA, Neves JEP. Escalas de avaliação em transtornos alimentares. Rev Psiquiat Clinica 1999; 26(1):41-47.

13. Organização Mundial da Saúde. Obesidade: prevenindo e controlando a epidemia global. São Paulo: Roca; 2004. 256 p.

14. Brasil. Conselho Nacional de Saúde. Resolução no 466, de 12 de dezembro de 2012. Diário Oficial da União 13 dez. 2012.

15. Monteiro MRP, Andrade MLO, Zanirati VF, Silva RR. Hábito e consumo alimentar de estudantes do sexo feminino dos cursos de nutrição e de enfermagem de uma Universidade Pública Brasileira. Revista APS 2009; 12(3):271-277.

16. Feitosa EPS, Dantas CAO, Andrade-Wartha ERS, Marcellini PS, Mendes-Neto RS. Hábitos alimentares de estudantes de uma universidade pública no nordeste, Brasil. Alim Nut Araraquara 2010; 21(2):225-230.

17. Castro JS, Miranda AS, Santana RF. Percepção de imagem corporal e consumo de fibras em acadêmicas de odontologia de instituição de ensino superior do sudoeste da Bahia. C\&D-Revista Eletrônica da Fainor 2015; 8(10):71-82.

18. Santos MA, Garcia RWD, Santos ML. A sujeição aos padrões corporais culturalmente construídos em mulheres de baixa renda. Demetra 2015; 10(4):761-774. 
19. Lopes LCC, Ruiz CS, Alves MS, Costa MEA, Mais SER, Marroni D. Autopercepção da imagem corporal entre universitários: uma análise comparativa. RBNE 2012; 6(34):325-332.

20. Wanden-Berghe C, Rodero HM, Martin AR, Ruiz JPN, Victoria EM, Valero JS et al. A calidade de vida y sus factores determinantes em universitários españoles de Ciencias de la Salud. Nutr Hosp. 2015; 31(2):952-958.

21. Garcia CA, Castro TG, Soares RM. Comportamento alimentar e imagem corporal entre estudantes de nutrição de uma universidade pública de Porto Alegre-RS. Rev HCPA 2010; 30(3):219-224.

22. Nilson G, Pardo ER, Rigo LC, Hallal PC. Espelho, espelho meu: um estudo sobre autoimagem corporal de estudantes universitários. Rev Bras Ativ Fis e Saúde 2013; 18(1):112-120.

23. Miranda VPN, Filgueiras JF, Neves CM, Teixeira PC, Ferreira MEC. Insatisfação corporal em universitários de diferentes áreas de conhecimento. J Bras Psiquiat. 2012; 61(1):25-32.

24. Graup S, Pereira EF, Lopes AS, Araújo VC, Legnani RS, Borgatto AF. Associação entre a percepção da imagem corporal e indicadores antropométricos de escolares. Rev. Bras. Educ. Fis. Esporte 2008; 22:129-38.

25. Pereira EF, Graup S, Lopes AS, Borgatto AF, Daronco LE. Percepção da imagem corporal de crianças e adolescentes com diferentes níveis sócio-econômicos na cidade de Florianópolis, Santa Catarina, Brasil. Rev Bras Saúde Matern Infant. 2009; 9:253-62.

26. Alvarenga MS, Philippi ST, Lourenço BH, Sato PM, Cagliusi FB. Insatisfação com a imagem corporal em universitárias brasileiras. J Bras Psiquiat. 2010; 59(1):44-51.

Recebido: 19/04/2016

Revisado: 22/10/2016

Aceito: 26/1/2017 